\section{Gicht-Patienten erreichen selten Therapieziele}

\author{
Alvarado-de la Barrera C et al. Are Target Urate \\ and Remission Possible in Severe Gout? A \\ Five-year Cohort Study. J Rheumatol 2020; 47: \\ 132-139
}

Wissenschaftlerinnen und Wissenschaftler der Gicht-Studiengruppe GRESGO bestimmten, wie viele Patientinnen und Patienten mit nicht schwerer und schwerer Gicht die Zielserumurat-Werte (SU) von < 6 bzw. $<5$ mg/dl erreichten, und bestimmten den Anteil der Patienten mit Remission nach 5 Jahren Nachbeobachtung.

Für ihre Analyse untersuchten sie die Studienteilnehmer der prospektiven Observationsstudie GRESGO in Abständen von 6 Monaten. Dokumentiert wurden die demografischen und klinischen Daten zu Studienbeginn. Während der Untersuchungstermine wurden Serumurat, Gichtanfälle und Belastungen mit Gichtknoten dokumentiert und die gesundheitsbezogene Lebensqualität (HRQOL) mit dem EQ-5D erfasst. Die Aktivitätsbeschränkungen wurden mit- hilfe des an die Gicht angepassten HAQFragebogens (Health Assessment Questionaire) beurteilt und die Schmerzen und die globale Einschätzung des Patienten mithilfe visueller Analogskalen bewertet. Die Behandlung von Gicht und der damit assoziierten Krankheiten, inklusive der medikamentösen Therapie, erfolgte gemäß den Leitlinien.

Die GRESGO-Studienkohorte bestand aus 500 Patientinnen und Patienten mit Gicht, davon waren $97 \%$ Männer. Von diesen hatten 221 eine schwere Gicht (44\%) und 279 eine nicht schwere Gicht (56\%). Eine schwere Gicht war definiert mit $\geq 5$ Gichtknoten und/oder intradermalen Knoten zu Studienbeginn.

Mononatriumurat-Kristalle in der Synovialflüssigkeit oder den Knoten wurden bei 67\% der Patientinnen und Patienten beobachtet. Studienteilnehmer mit schwerer und nicht schwerer Gicht unterschieden sich signifikant. So waren die Patientinnen und Patienten mit schwerer Gicht signifikant jünger zum Krankheitsbeginn, hatten einen geringeren Bildungsgrad und sozioökonomischen Status, waren länger krank und versäumten öfter die Laborkontrollen. Darüber hinaus litten sie häufiger an Bluthochdruck sowie chronischem Nierenversagen. Die Gruppe mit schwerer Gicht hatte signifikant mehr betroffene Gelenke, mehr Gichtknoten, deutlich mehr Aktivitätsbeschränkungen und einen geringeren HRQOL als die Gruppe mit nicht schwerer Gicht. Die Patientinnen und Patienten mit schwerer Gicht erhielten höhere Allopurinoldosen als diejenigen mit nicht schwerer Gicht und dies auch im Verlauf der Nachbeobachtungsperiode.

Hinsichtlich der Anteile derjenigen, die die Zielwerte von $<6$ bzw. $<5$ mg/dl erreichten, ergaben sich keine signifikanten Unterschiede zwischen den Gruppen. Der höchste Anteil der Patientinnen und Patienten, die den tiefsten Wert des Serumurat-Wertes erreichten, wurden nach 3-4 Jahren Nachuntersuchung gefunden. Im fünften Jahr nahmen diese Anteile ab. Nach einem Jahr waren 9,1\% der Patientinnen und $\mathrm{Pa}$ tienten in Remission, nach 2 Jahren 30 und $28 \%$ nach 3 Jahren. Der höchste Anteil wurde mit $39 \%$ nach 4 Jahren Nachbeobachtung erreicht, da waren allerdings nur noch wenige Teilnehmer (insgesamt 81) in der Studie verblieben. Nach 5 Jahren waren dies nur noch 40 (19 in der schwerer GichtGruppe und 21 in der nicht schweren GichtGruppe). Keiner der Patienten mit schwerer Gicht erreichte eine Remission.

FAZIT

Patientinnen und Patienten mit schwerer Gicht erreichen die Zielserumurat-Werte nur schwer und eine Remission gar nicht, so die GRESGO-Studiengruppe. Die Haupthindernisse für die Erreichung des Ziel-SU und der Gicht-Remission sind eine schlechte MedikamentenCompliance, persistierende Gichtknoten und mangelhafte Adhärenz.

Richard Kessing, Zeiskam 